DESY 06-014

\title{
Moduli-Induced Gravitino Problem
}

\author{
Motoi Endo ${ }^{1,2}$, Koichi Hamaguchi ${ }^{1}$ and Fuminobu Takahashi ${ }^{1,2}$ \\ 1 Deutsches Elektronen Synchrotron DESY, Notkestrasse 85, 22607 Hamburg, Germany \\ 2 Institute for Cosmic Ray Research, University of Tokyo, Chiba 277-8582, Japan
}

\begin{abstract}
We investigate the cosmological moduli problem by studying a modulus decay in detail and find that the branching ratio of the gravitino production is generically of $O(0.01-1)$, which causes another cosmological disaster. Consequently, the cosmological moduli problem cannot be solved simply by making the modulus mass heavier than $100 \mathrm{TeV}$. We also illustrate our results by explicitly calculating the branching ratio into the gravitinos in the mixed modulus-anomaly/KKLT- and racetrack-type models.
\end{abstract}

The cosmological moduli problem [1] is one of the most challenging puzzles in particle physics and cosmology. In this letter, we show that the problem is even more difficult than usually thought.

In supergravity/superstring theories, generically there exist moduli fields which have flat potentials and obtain masses from supersymmetry (SUSY) breaking and nonperturbative effects. During an inflationary period, a modulus field $X$ is likely to develop a large expectation value. After the end of the inflation, it starts a coherent oscillation and soon dominates the energy density of the universe. Due to the interaction suppressed by the Planck scale $M_{P}=2.4 \times 10^{18} \mathrm{GeV}$, the decay rate of the modulus $X$ is extremely small:

$$
\Gamma_{X}=\frac{c}{4 \pi} \frac{m_{X}^{3}}{M_{P}^{2}},
$$

which leads to an onset of a radiation-dominated universe with a very low temperature:

$$
\begin{aligned}
T_{X} & =\left(\pi^{2} g_{*} / 90\right)^{-1 / 4} \sqrt{M_{P} \Gamma_{X}} \\
& \simeq 5.5 \times 10^{-3} \mathrm{MeV} \cdot c^{\frac{1}{2}}\left(\frac{m_{X}}{1 \mathrm{TeV}}\right)^{3 / 2}
\end{aligned}
$$

Here, $c$ is an order one coefficient and $g_{*}$ is the effective number of massless degrees of freedom. This is cosmologically unacceptable because a successful big-bang nucleosynthesis $(\mathrm{BBN})$ requires that the (last) radiationdominated universe starts with temperature higher than $\sim 5 \mathrm{MeV}$ 2].

As is clear from Eq. (2), a simple solution would be to assume that the modulus $X$ is ultra heavy ${ }^{\mathrm{a}}$ :

$$
m_{X} \gtrsim 100 \mathrm{TeV} \quad \rightarrow \quad T_{X} \gtrsim \mathcal{O}(\mathrm{MeV})
$$

Actually, there have been proposed scenarios with such a large modulus mass (cf. [5, 6, 7, 8, 9]).

However, there exists yet another serious cosmological obstacle even for heavy moduli scenarios. The new problem is caused by the gravitino which is produced by the modulus decay. Indeed, as we will show, the branching

\footnotetext{
a See Refs. 3, 4] for other solutions.
}

ratio of the modulus decay into the gravitino is generically quite large

$$
B_{3 / 2} \equiv \operatorname{Br}(X \rightarrow \text { gravitino })=\mathcal{O}(0.01-1),
$$

which causes serious problems after the modulus decay. We call this problem the moduli-induced gravitino problem.

The gravitino production via modulus decay and its cosmological implications have been previously discussed in Refs. 10, 11], taking $\operatorname{Br}(X \rightarrow$ gravitino $) \ll 1$. The main purpose of this letter is to show that Eq. (4) holds in a generic setup, and to emphasize how disastrous its consequences are. We also exemplify explicit results in the mixed modulus-anomaly/KKLT mediation [6, 7] and in the racetrack [8] setups.

Let us first estimate the branching ratio of a modulus decay into gravitino(s). We consider a heavy modulus scenario, $m_{X} \gtrsim 100 \mathrm{TeV}$ [cf. Eq. (3)]. On the other hand, the gravitino is likely to be (much) lighter than $100 \mathrm{TeV}$, because too large gravitino mass requires a finetuning in the Higgs sector due to the anomaly-mediated effects. Thus, we assume $m_{X} \gg m_{3 / 2}$ hereafter. After choosing the unitary gauge in the Einstein frame, where the goldstino component is completely absorbed into the gravitino under the super-Higgs mechanism 12], the relevant gravitino-modulus couplings are 10, 11, 13]

$$
\begin{aligned}
e^{-1} \mathcal{L}= & -\frac{1}{8} \epsilon^{\mu \nu \rho \sigma}\left(G_{X} \partial_{\rho} X-G_{\bar{X}} \partial_{\rho} \bar{X}\right) \bar{\psi}_{\mu} \gamma_{\nu} \psi_{\sigma} \\
& -\frac{1}{8} e^{G / 2}\left(G_{X} X+G_{\bar{X}} \bar{X}\right) \bar{\psi}_{\mu}\left[\gamma^{\mu}, \gamma^{\nu}\right] \psi_{\nu},
\end{aligned}
$$

where $\psi_{\mu}$ is the gravitino field. Here and hereafter, we set $M_{P}=1$ unless explicitly written. The function $G=$ $K+\ln |W|^{2}$ is the total Kähler potential, and $G_{i}$ denotes a derivative of $G$ with respect to the field $i$. The real and imaginary components of $X=\left(X_{R}+i X_{I}\right) / \sqrt{2}$ have the decay rate ${ }^{\mathrm{b}}$,

$$
\Gamma\left(X_{R, I} \rightarrow 2 \psi_{3 / 2}\right) \simeq \frac{1}{288 \pi} \frac{\left|G_{X}\right|^{2}}{g_{X \bar{X}}} \frac{m_{X}^{5}}{m_{3 / 2}^{2}},
$$

\footnotetext{
b The branching ratio of the single gravitino production is suppressed due to the phase space; $\operatorname{Br}\left(X \rightarrow \tilde{X} \psi_{3 / 2}\right) \lesssim$ $\left(m_{3 / 2} / m_{X}\right)^{2}$.
} 
in the limit of $m_{X} \gg m_{3 / 2}$ after the canonical normalization, $\hat{X}=\sqrt{g_{X \bar{X}}} X$, where $g_{i j^{*}}=K_{i j^{*}}$ is the Kähler metric. We emphasize that the gravitino mass in the denominator arises from the longitudinal component of the gravitino, which corresponds to the goldstino, $\psi_{\mu} \sim\left(p_{\mu} / m_{3 / 2}\right) \chi_{G S}$, in the massless limit. This factor magnifies the decay rate by a factor of $\sim\left(m_{X} / m_{3 / 2}\right)^{4}$, compared to the previous results [10, 11, 14].

The auxiliary field of the modulus, $G_{X}$, represents its fractional contribution to the total amount of the SUSY breaking. It may be small enough to suppress the gravitino-production rate. However, we can see that in the framework of the $4 \mathrm{D} N=1$ supergravity, $G_{X}$ is generically bounded below, $G_{X} \gtrsim m_{3 / 2} / m_{X} \equiv R^{-1}$. The scalar potential is given by $V=e^{G}\left(G^{i} G_{i}-3\right)$ and the (almost) vanishing vacuum energy demands that at least one of the auxiliary fields, $G_{i}$, should take $G^{i} G_{i} \sim O(1)$, where $G^{i}=g^{i j^{*}} G_{j^{*}}$. If the modulus field plays the role, it dominantly decays into the gravitino [cf. Eqs.(11) and [6)] and hence clearly $B_{3 / 2} \simeq 1$. Instead, by introducing a hidden sector field $Z$ with $G_{Z} \sim O(1)$, the modulusauxiliary field $G_{X}$ can be small ${ }^{\mathrm{c}}$. In the following discussion, we assume that the modulus field does not decay into the hidden-sector field for simplicity. In order to see how small $G_{X}$ can be, we investigate the condition to minimize the potential, $V_{X}=0$, leading to

$$
G^{X} \nabla_{X} G_{X}+G^{Z} \nabla_{X} G_{Z}+G_{X}=0,
$$

where $\nabla_{i} G_{j}=G_{i j}-\Gamma_{i j}^{k} G_{k}$ with the connection, $\Gamma_{i j}^{k}=$ $g^{k \ell^{*}} g_{i \ell^{*} j}$. We assume that the Kähler potential includes no enhancement factor, and especially $g_{X \bar{X}}, g_{Z \bar{Z}} \sim$ 1 and $K_{X} \sim 1$. The first term in Eq. (7) then becomes $\sim G^{X} R$, because $m_{X} / m_{3 / 2} \sim\left|\nabla_{X} G_{X}\right|+$ $\mathcal{O}\left(G_{i}\right)$. Barring cancellation, the second term is given by $\max \left[W_{Z} / W, W_{X Z} / W, K_{X Z}, \Gamma_{X Z}^{Z}\right]$, which is $O(1)$ unless the hidden sector takes a special form. Thus, we arrive at $\left|G_{X}\right| \sim R^{-1}$ for $\left|g^{X \bar{Z}}\right|<R^{-1}$ and $\left|G_{X}\right| \gtrsim R^{-1}$ for $\left|g^{X \bar{Z}}\right| \gtrsim R^{-1}$. As a result, Eq. (6) becomes

$$
\Gamma\left(X_{R, I} \rightarrow 2 \psi_{3 / 2}\right) \simeq \frac{|\kappa|^{2}}{288 \pi} \frac{1}{g_{X \bar{X}}} m_{X}^{3},
$$

where we define $G_{X}=\kappa / R=\kappa\left(m_{3 / 2} / m_{X}\right)$ with $|\kappa| \gtrsim 1$. Note that the above discussion is valid for any value of $m_{X}$, as long as $R \gg 1$ is satisfied.

The modulus field also decays into radiation, that is, the standard model (SM) particles and their superpartners. The relevant interactions of $X$ then stem from the dilatonic coupling with the gauge sector, $\int d^{2} \theta X W W$,

\footnotetext{
c The potential may be up-lifted by adding the $D$-term, $V^{(D)}$. One can show that $\left|G_{X}\right| \gtrsim R^{-1}$ also in this case, as far as $V_{X}^{(D)} / V^{(D)} \sim O(1)$, which corresponds to the second term in
Eq. (7).
}

leading to

$$
\begin{aligned}
e^{-1} \mathcal{L}= & -\frac{1}{4 \sqrt{g_{X \bar{X}}}}\left[\frac{\hat{X}_{R}}{\left\langle X_{R}\right\rangle} F_{\mu \nu}^{(a)} F^{(a) \mu \nu}+\frac{\hat{X}_{I}}{\left\langle X_{R}\right\rangle} F_{\mu \nu}^{(a)} \widetilde{F}^{(a) \mu \nu}\right. \\
& \left.-\frac{\sqrt{2}}{\left\langle X_{R}\right\rangle} e^{G / 2}\left(G^{\bar{X}}{ }_{X} \hat{X} \bar{\lambda}^{(a)} \mathcal{P}_{R} \lambda^{(a)}+\text { h.c. }\right)\right]
\end{aligned}
$$

after the canonical normalization, where $F_{\mu \nu}$ and $\lambda$ are the field strength of the gauge boson and its superpartner with a generator index $a$ of the corresponding gauge symmetry. The chiral projection operators are defined as $\mathcal{P}_{R, L}=\left(1 \pm \gamma_{5}\right) / 2$. We notice $e^{G / 2}\left|G_{X}^{\bar{X}_{X}}\right|$ is approximately given by the modulus mass for $R \gg 1$. Then the decay rate is

$$
\Gamma\left(X_{R, I} \rightarrow \text { radiation }\right) \simeq \frac{3}{8 \pi} \frac{1}{g_{X \bar{X}}} \frac{m_{X}^{3}}{\left\langle X_{R}\right\rangle^{2}},
$$

for $S U(3)_{c} \times S U(2)_{L} \times U(1)_{Y}$, corresponding to $c=O(1)$. We notice that a half of the decay rate comes from the channel of the gaugino production, in contrast to the results of Refs. 11, 14]. From Eqs. (8) and (10), we obtain the branching ratio of the gravitino production as

$$
\operatorname{Br}\left(X_{R, I} \rightarrow 2 \psi_{3 / 2}\right) \simeq \frac{|\kappa|^{2}\left\langle X_{R}\right\rangle^{2} / 108}{1+|\kappa|^{2}\left\langle X_{R}\right\rangle^{2} / 108}
$$

It is important to note that the production rate of the gravitino channel is one of the dominant processes in the modulus decay. Actually, it becomes an order of $0.01-1$.

The other decay processes are suppressed, except for a possible decay into the Higgs(-ino) [14]. However, this decay channel is model-dependent, and does not change the above result $c=O(1)$.

Let us show how such large branching ratio into the gravitinos jeopardizes the success of the standard big bang cosmology. We consider the constraints from (i) the speed-up effect, (ii) BBN, and (iii) the lightest SUSY particle (LSP) abundance. First let us consider the socalled speed-up effect which modifies mostly the ${ }^{4} \mathrm{He}$ abundance. This sets a bound on the abundance of the gravitinos with $m_{3 / 2} \lesssim 20 \mathrm{TeV}$ (cf. [15]), since they decay after the neutron-proton transformation decouples. The observational data put an upper bound on the ratio of the energy densities of the gravitinos to the standard model particles at the BBN epoch [16] as

$$
\left.f_{3 / 2} \equiv \frac{\rho_{3 / 2}}{\rho_{S M}}\right|_{\mathrm{BBN}} \lesssim 0.2, \quad(95 \% \text { C.L. }) .
$$

In our heavy moduli scenario, however, the ratio is bounded below: $f_{3 / 2} \geq B_{3 / 2} /\left(1-B_{3 / 2}\right)$, where the equality holds if the gravitinos are still relativistic at the BBN epoch and most of the superpartner of the SM particles directly produced from the modulus decay soon annihilate into the SM particles. Thus we obtain

$$
B_{3 / 2}<0.2,
$$


irrespective of whether the gravitino is stable or unstable. For $B_{3 / 2}>0.2$, the gravitinos from the modulus decay always upset the standard BBN, as long as $m_{3 / 2} \lesssim 20 \mathrm{TeV}$.

Now we discuss the cases of the stable and unstable gravitinos separately. First we take up the unstable gravitinos, which is the case if the gravitino mass is heavier than the LSP mass, $m_{3 / 2}>m_{\mathrm{LSP}}$. The gravitino-toentropy ratio is given by

$$
\begin{aligned}
Y_{3 / 2} & \equiv \frac{n_{3 / 2}}{s} \simeq 2 B_{3 / 2} \frac{3 T_{X}}{4 m_{X}} \\
& \simeq 2.6 \times 10^{-7} c^{\frac{1}{2}} B_{3 / 2}\left(\frac{m_{X}}{10^{3} \mathrm{TeV}}\right)^{\frac{1}{2}} .
\end{aligned}
$$

The BBN severely constrains $Y_{3 / 2}$ [15, 17]. Even if we adopt the recent analysis on ${ }^{4} \mathrm{He}$ abundance which has taken account of possible large systematic error [18], $Y_{3 / 2}$ must be smaller than $2 \times 10^{-12}$ at $95 \%$ C.L. for $m_{3 / 2} \simeq 30 \mathrm{TeV}$ [15], and the bound becomes much severer for smaller $m_{3 / 2}$. Therefore the branching ratio into the gravitinos must be extremely small:

$$
B_{3 / 2}<10^{-5} \epsilon c^{-\frac{1}{2}}\left(\frac{10^{3} \mathrm{TeV}}{m_{X}}\right)^{\frac{1}{2}}
$$

for $m_{\mathrm{LSP}}<m_{3 / 2} \leq 30 \mathrm{TeV}$. Here $\epsilon \leq 1$ parameterizes the BBN bound: $\epsilon=1$ for $m_{3 / 2} \simeq 30 \mathrm{TeV}$, and $10^{-5} \lesssim$ $\epsilon \ll 1$ for $m_{3 / 2}<30 \mathrm{TeV}$. In addition, the abundance of the LSPs from the gravitino decay is (cf. [19])

$$
\left.Y_{\mathrm{LSP}}\right|_{\psi_{3 / 2}} \simeq \min \left[Y_{3 / 2}, \sqrt{\frac{45}{8 \pi^{2} g_{*}}} \frac{1}{M_{P} T_{3 / 2}\left\langle\sigma_{a n n} v\right\rangle}\right],
$$

where $\left\langle\sigma_{a n n} v\right\rangle$ is the thermally averaged annihilation cross section of the LSP, and $T_{3 / 2}$ is the decay temperature of the gravitino. Since the LSP abundance must be smaller than the dark matter abundance, we have another constraint on $B_{3 / 2}$ :

$B_{3 / 2}<1.8 \times 10^{-5} c^{-\frac{1}{2}}\left(\frac{100 \mathrm{GeV}}{m_{\mathrm{LSP}}}\right)\left(\frac{\Omega_{\mathrm{DM}} h^{2}}{0.13}\right)\left(\frac{10^{3} \mathrm{TeV}}{m_{X}}\right)^{\frac{1}{2}}$

for $m_{3 / 2}>m_{\mathrm{LSP}}$. Here $\Omega_{\mathrm{DM}}$ is the density parameter of the dark matter, $h$ is the present Hubble parameter in units of $100 \mathrm{~km} / \mathrm{sec} / \mathrm{Mpc}$, and we have assumed $\left\langle\sigma_{a n n} v\right\rangle<10^{-6} \mathrm{GeV}^{-2}$ and $m_{3 / 2}<100 \mathrm{TeV}$. We can see that (13), (15) and (17) rule out the unstable gravitinos, unless $B_{3 / 2}$ is extraordinarily small in spite of our result $B_{3 / 2}=O(0.01-1)$.

Next we consider the stable gravitinos, which is the case if the gravitino is the LSP. A constraint then comes from the requirement that the gravitino abundance should not exceed the dark matter abundance, and we only have to replace $m_{\mathrm{LSP}}$ with $m_{3 / 2}$ in (17):

$B_{3 / 2}<1.8 \times 10^{-2} c^{-\frac{1}{2}}\left(\frac{100 \mathrm{MeV}}{m_{3 / 2}}\right)\left(\frac{\Omega_{D M} h^{2}}{0.13}\right)\left(\frac{10^{3} \mathrm{TeV}}{m_{X}}\right)^{\frac{1}{2}}$

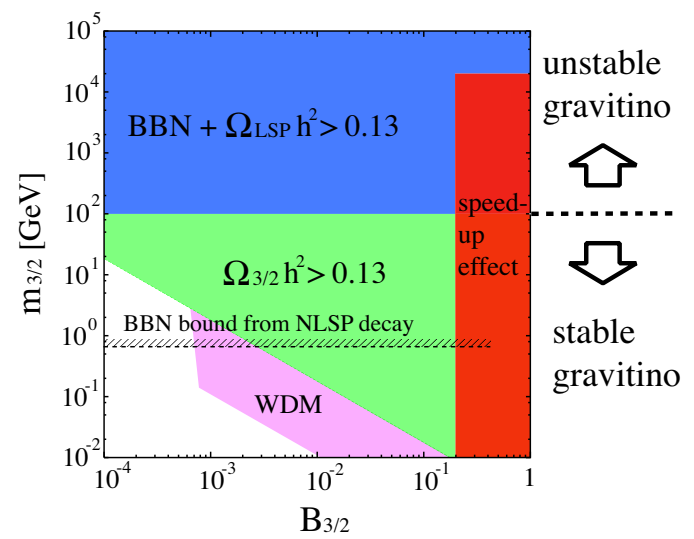

FIG. 1: The cosmological bounds on $m_{3 / 2}$ and $B_{3 / 2}$. Shaded regions are excluded by cosmological arguments. See the text for details. The horizontal dashed line denotes the BBN bound from the stau NLSP decay into gravitinos for $m_{\mathrm{NLSP}}=100 \mathrm{GeV}$. We have chosen $m_{X}=10^{3} \mathrm{TeV}$ and $c=1$ as representative values. The bounds become severer for larger $m_{X}$.

for the stable gravitino. Furthermore, the bound on the gravitino abundance is severer by an order of magnitude for $m_{3 / 2} \lesssim 100 \mathrm{MeV}\left(m_{X} / 10^{3} \mathrm{TeV}\right)^{-1 / 2}$, due to the present large free-streaming velocity [20]. See Fig. 1] From (13) and (18), we conclude that large $B_{3 / 2}$ encounters trouble even for the stable gravitinos.

Lastly we comment on the lightest superpartner of the SM particles, denoted by $\chi$, produced from the modulus decay through the gauginos. From the discussion above, the number of $\chi$ generated from the decay of unit quantum of the modulus field is order unity. If $\chi$ is the LSP and stable, it must be electrically neutral. To satisfy $\Omega_{\mathrm{LSP}} h^{2}<0.13$, the pair annihilation cross section must be large [14, 19], which in turn constrains the mass spectrum of the SUSY particles. In the case of the gravitino LSP, there is a strict BBN bound on the abundance and lifetime of the next-to-LSP (NLSP) $\chi$. For a stau NLSP $\tilde{\tau}$, we find upper bounds on the gravitino mass, $m_{3 / 2} \lesssim(0.3-1) \mathrm{GeV}$ for $m_{X}=10^{3} \mathrm{TeV}$ and $100 \mathrm{GeV} \lesssim m_{\tilde{\tau}} \lesssim 1 \mathrm{TeV}^{\mathrm{d}}$. For a neutralino NLSP, the bound becomes severer.

We summarize the bounds considered above in Fig. 1 from which we can see how serious the cosmological moduli problem becomes as a result of our finding that $B_{3 / 2}$ should be $O(0.01-1)$. Note that the bounds on $B_{3 / 2}$ [cf. [15), (17) and [18)] become severer for heavier modulus mass and larger $c$, although the dependence is weak.

We have used $Y_{\tilde{\tau}} \simeq\left(45 / 8 \pi^{2} g_{*}\right)^{1 / 2}\left(M_{P} T_{X}\left\langle\sigma_{a n n} v\right\rangle\right)^{-1}$ [19] with $\left\langle\sigma_{a n n} v\right\rangle \lesssim 10^{-3} m_{\tilde{\tau}}^{-2} 21$, BBN bounds from 15] with $\tilde{\tau}^{\prime}$ s hadronic branching in [22], and $\tau_{\tilde{\tau}} \simeq$ $6 \sec \left(m_{\tilde{\tau}} / 100 \mathrm{GeV}\right)^{-5}\left(m_{3 / 2} / 10 \mathrm{MeV}\right)^{2}$. The bound from $\Omega_{3 / 2}$ (from $\tilde{\tau})<\Omega_{\mathrm{DM}}$ is slightly weaker than the BBN bound. 
Let us explicitly calculate the branching fraction of the gravitino in some models. The relevant terms in the Lagrangian of the mixed modulus-anomaly/KKLT mediation model [6, 7] are given by

$$
\begin{aligned}
& f=-3(X+\bar{X})^{n / 3}+C_{Z}(X+\bar{X})^{l}|Z|^{2}, \\
& W=W_{0}+a e^{-b X}+W_{\text {Hidden }}(Z),
\end{aligned}
$$

where the Kähler potential is defined as $f=-3 e^{-K / 3}$. Here coefficients $b$ and $C_{Z}$ are real with $b X_{R} \gg 1$, while $a$ and $W_{0}$ are complex. Then the modulus mass is

$$
m_{X}^{2} \simeq 2\left(b X_{R}\right)^{2} m_{3 / 2}^{2} .
$$

The gravitino mass $m_{3 / 2}$ is much larger than the soft mass scale []. On the other hand, $G_{X}$ is

$$
G_{X} \simeq-\frac{2 n+3 l}{2 X_{R}} \frac{1}{b X_{R}}
$$

where we assumed $\langle Z\rangle \ll 1$ and the vanishing cosmological constant. Thus the branching ratio becomes

$$
\operatorname{Br}\left(X_{I} \rightarrow 2 \psi_{3 / 2}\right) \simeq \frac{(2 n+3 l)^{2} / 216}{1+(2 n+3 l)^{2} / 216} .
$$

In the original KKLT model [ 6 ] with the lift-up potential due to $\overline{D 3}$ brane, which is realized by choosing $n=3$ and $l=0$, the branching ratio is $B_{3 / 2} \simeq 0.14$.

In the racetrack-type models [8], the Kähler potential is the same as Eq. (19) but the superpotential is

$$
W=a_{1} e^{-b_{1} X}+a_{2} e^{-b_{2} X}+W_{\text {Hidden }}(Z),
$$

where $a_{i}$ 's are complex and $b_{i}$ 's are real numbers. Here $b_{i} X_{R} \gg 1$. The modulus mass and $G_{X}$ are

$$
m_{X}^{2} \simeq \frac{4}{n^{2}}\left(b_{1} X_{R}\right)^{2}\left(b_{2} X_{R}\right)^{2} m_{3 / 2}^{2}
$$

$$
G_{X} \simeq-\frac{n(2 n+3 l)}{2 \sqrt{2} X_{R}} \frac{1}{\left(b_{1} X_{R}\right)\left(b_{2} X_{R}\right)}
$$

Therefore the branching ratio becomes the same as Eq. (23). For $n=1$ and $l=0$, one obtains $B_{3 / 2} \simeq 0.018$.

In summary, we have shown that the branching ratio of the modulus decay into gravitinos is $O(0.01-1)$, and hence heavy modulus scenario is plagued with the moduli-induced gravitino problem. Here let us comment on possible ways out. Unless either $B_{3 / 2}$ or the gravitino mass is extremely small, there is no way to circumvent the problem other than introducing something that dilutes the modulus field and the subsequently produced gravitinos and (N)LSPs. One of the candidates is the thermal inflation [23]. Another is Q-balls [24] in the Affleck-Dine mechanism [25]. In the latter case, the Q-balls can not only dilute the unwanted gravitinos but also generate the baryon asymmetry successfully. Detailed discussion is beyond the scope of this letter and will be presented elsewhere [26].

We thank Wilfried Buchmüller for comments. M.E. is grateful to Shuntaro Nakamura and Masahiro Yamaguchi for private communication. M.E. and F.T. would like to thank the Japan Society for Promotion of Science for financial support.

Note Added: Shortly after our letter, Ref. 27] appeared, pointing out the same problem. They also discussed the gravitino much heavier than $100 \mathrm{TeV}$ as a possible solution to the moduli-induced gravitino problem. More recently, Ref. 28] has pointed out $G_{X}$ can be much smaller than $R^{-1}$ in some situations. However, for general non-renormalizable couplings in $K$, our estimate on the gravitino production still holds. See [29] for details.
[1] G. D. Coughlan, W. Fischler, E. W. Kolb, S. Raby and G. G. Ross, Phys. Lett. B 131 (1983) 59; T. Banks, D. B. Kaplan and A. E. Nelson, Phys. Rev. D 49 (1994) 779; B. de Carlos, J. A. Casas, F. Quevedo and E. Roulet, Phys. Lett. B 318 (1993) 447.

[2] M. Kawasaki, K. Kohri and N. Sugiyama, Phys. Rev. Lett. 82, 4168 (1999); Phys. Rev. D 62, 023506 (2000); S. Hannestad, Phys. Rev. D 70, 043506 (2004); K. Ichikawa, M. Kawasaki and F. Takahashi, Phys. Rev. D 72, 043522 (2005).

[3] M. Dine, Y. Nir and Y. Shadmi, Phys. Lett. B 438, 61 (1998); A. D. Linde, Phys. Rev. D 53, 4129 (1996); L. Randall and S. D. Thomas, Nucl. Phys. B 449, 229 (1995); D. H. Lyth and E. D. Stewart, Phys. Rev. D 53, 1784 (1996); M. Kawasaki and F. Takahashi, Phys. Lett. B 618, 1 (2005).

[4] J. Yokoyama, arXiv hep-ph/0601067

[5] L. Randall and R. Sundrum, Nucl. Phys. B557, 79 (1999); G.F. Giudice, M.A. Luty, H. Murayama and
R. Rattazzi, JHEP 9812, 027 (1998); J.A. Bagger, T. Moroi and E. Poppitz, JHEP 0004, 009 (2000).

[6] S. Kachru, R. Kallosh, A. Linde and S.P. Trivedi, Phys. Rev. D68, 046005 (2003).

[7] K. Choi, A. Falkowski, H.P. Nilles, M. Olechowski and S. Pokorski, JHEP 0411 (2004) 076; K. Choi, A. Falkowski, H. P. Nilles and M. Olechowski, Nucl. Phys. B 718, 113 (2005); M. Endo, M. Yamaguchi and K. Yoshioka, Phys. Rev. D 72, 015004 (2005); K. Choi, K. S. Jeong and K. i. Okumura, JHEP 0509, 039 (2005); A. Falkowski, O. Lebedev and Y. Mambrini, JHEP 0511, 034 (2005).

[8] N.V. Krasnikov, Phys. Lett. B193 37 (1987); T.R. Taylor, Phys. Lett. B252 59 (1990); J.A. Casas, Z. Lalak, C. Munoz and G.G. Ross, Nucl. Phys. B347 243 (1990); B. de Carlos, J.A. Casas and C. Munoz, Nucl. Phys. B399 623 (1993).

[9] W. Buchmuller, K. Hamaguchi, O. Lebedev and M. Ratz, Nucl. Phys. B 699, 292 (2004). 
[10] M. Hashimoto, K. I. Izawa, M. Yamaguchi and T. Yanagida, Prog. Theor. Phys. 100, 395 (1998).

[11] K. Kohri, M. Yamaguchi and J. Yokoyama, Phys. Rev. D 72 (2005) 083510; Phys. Rev. D 70 (2004) 043522.

[12] E. Cremmer, B. Julia, J. Scherk, S. Ferrara, L. Girardello and P. van Nieuwenhuizen, Nucl. Phys. B 147, 105 (1979).

[13] J. Wess and J. Bagger, Supersymmetry and Supergravity, (Princeton Unversity Press, 1992).

[14] T. Moroi and L. Randall, Nucl. Phys. B 570, 455 (2000).

[15] K. Kohri, T. Moroi and A. Yotsuyanagi, arXiv hep-ph/0507245

[16] R. H. Cyburt, B. D. Fields, K. A. Olive and E. Skillman, Astropart. Phys. 23, 313 (2005).

[17] M. Kawasaki, K. Kohri and T. Moroi, Phys. Lett. B 625, 7 (2005); Phys. Rev. D 71, 083502 (2005).

[18] K. A. Olive and E. D. Skillman, Astrophys. J. 617, 29 (2004).

[19] M. Fujii and K. Hamaguchi, Phys. Lett. B 525, 143 (2002); Phys. Rev. D 66, 083501 (2002).
[20] K. Jedamzik, M. Lemoine and G. Moultaka, arXiv astro-ph/0508141

[21] T. Asaka, K. Hamaguchi and K. Suzuki, Phys. Lett. B 490, 136 (2000).

[22] J. L. Feng, S. f. Su and F. Takayama, Phys. Rev. D 70, 063514 (2004).

[23] D. H. Lyth and E. D. Stewart, in Ref. 3].

[24] S. R. Coleman, Nucl. Phys. B 262, 263 (1985) [Erratumibid. B 269, 744 (1986)].

[25] I. Affleck and M. Dine, Nucl. Phys. B 249, 361 (1985); M. Dine, L. Randall and S. Thomas, Nucl. Phys. B 458, 291 (1996).

[26] M. Endo, K. Hamaguchi and F. Takahashi, in preparation.

[27] S. Nakamura and M. Yamaguchi, arXiv hep-ph/0602081

[28] M. Dine, R. Kitano, A. Morisse and Y. Shirman, arXiv hep-ph/0604140

[29] M. Endo, K. Hamaguchi and F. Takahashi, arXiv hep-ph/0605091 